\title{
Inexpensive Re-Configurable Process Simulator for the Feedback Control Lab
}

\author{
Robert Lynn Mueller \\ The Pennsylvania State University \\ New Kensington Campus
}

\begin{abstract}
A variety of control concepts and techniques must be covered in a comprehensive course on feedback controls. This need for variety imposes severe demands on laboratory facilities if they are to demonstrate an appropriate range of practical control exercises using all or most of the techniques. This problem has been minimized through the development of a re-configurable process simulator for a senior-level feedback control class in electro-mechanical engineering technology.
\end{abstract}

This paper describes a process simulator that is built from linear circuit components. It simulates the transfer functions of typical processes that would be encountered in an industrial environment. The parameters of the transfer function can be easily changed allowing the simulator to simulate a variety of possible process situations.

It can be used to illustrate the procedures involved in calculating PID parameters in the classroom as well as determining "in the field". The simulator then can be used to test the results and thereby give the student experience in tuning PID controllers. It can be used to verify the root locus and frequency domain calculations for various compensators such as lead, lag, and lead/lag. It can be also used to investigate steady-state error and process stability.

\section{Problems with Other Simulators}

How to demonstrate control concepts in the lab is an age-old problem. While all of these concepts can be demonstrated using a software simulator such as Matlab, nothing beats being able to physically change a setpoint and observe what happens to the process variable on a chart recorder.

There are many commercially available simulators for the control of flow, temperature, etc. These simulators are nice in that they provide the student the opportunity to actually see the process variable change as well as get exposure to the operation of field transmitters. However, they do not offer the ability to change the system's parameters and are, therefore, somewhat limited as to the variety of control concepts that can be demonstrated. They are also expensive and often bulky leading to storage and/or handling problems. 


\section{Design Goals}

To solve these problems, a simulator was designed that allows certain system parameters to be varied so that a wide variety of control concepts can be demonstrated. The primary design goal was that the simulator had to be cheap and easy to use. It was decided that a maximum number of control concepts could be demonstrated if the device could simulate both type 0 and type 1 systems. In addition, the plant transfer function $\mathrm{Gp}(\mathrm{s})$ had to be easily isolated for open loop tests, and the ability to close the loop had to also be included.

\section{Derivation of the Circuit}

The basis for the simulator lies in the fact that differential equations can be solved/simulated using an analog computer. Using this fact, a circuit for simulating type 0 and type 1 systems can be derived.

For a type 0 system,

$$
\mathrm{Gp}(\mathrm{s})=\mathrm{k} /\left(\mathrm{s}^{\wedge} 2+\mathrm{a} \mathrm{s}+\mathrm{b}\right)
$$

Since $G p(s)$ represents the output $Y(s)$ divided by the input $R(s)$,

or

$$
\mathrm{Y}(\mathrm{s}) / \mathrm{R}(\mathrm{s})=\mathrm{k} /\left(\mathrm{s}^{\wedge} 2+\mathrm{a} \mathrm{s}+\mathrm{b}\right)
$$

$$
\mathrm{Y}(\mathrm{s})\left(\mathrm{s}^{\wedge} 2+\mathrm{a} \mathrm{s}+\mathrm{b}\right)=\mathrm{k} \mathrm{R}(\mathrm{s})
$$

Taking the inverse Laplace transform,

or

$$
y^{\prime \prime}(t)+a y^{\prime}(t)+b y(t)=k r(t)
$$

$$
y^{\prime \prime}(t)=k r(t)-a y^{\prime}(t)-b y(t)
$$

This differential equation is simulated using the analog circuitry shown in Figure 1.

For a type 1 system,

$$
\mathrm{Gp}(\mathrm{s})=\mathrm{k} / \mathrm{s}\left(\mathrm{s}^{\wedge} 2+\mathrm{a} \mathrm{s}+\mathrm{b}\right)
$$

Using the same steps as above, it can be shown that

$$
y^{\prime \prime \prime}(t)=k r(t)-a y^{\prime \prime}(t)-b y^{\prime}(t)
$$

This differential equation is simulated using the analog circuitry shown in Figure 2 
Examining the similarities between the two circuits shows that the same hardware can represent both systems, the only difference being where the value of $y(t)$ is extracted. Figure 3 shows how the combined circuits are used to simulate a unity feedback control system for both a type 0 and a type 1 system. The process variable (PV) is $\mathrm{y}(\mathrm{t})$, and the input is the desired setpoint (SP).

The validity of this theoretical circuit was verified by comparing the Electronic Workbench simulation, of several $\mathrm{Gp}(\mathrm{s})$ plant transfer functions with the corresponding Matlab simulations.

\section{Description of Circuit}

The simulator circuit is shown in Figure 4.

Banana jacks are provided for the simulator inputs and outputs. Power is applied on the +15 Volts, -15 Volts, and ground jacks. The SP voltage is applied between the Input and Ground jacks. The PV is measured between the Output and Ground jacks.

Referring to Figure 3, Op amp "A" forms the error signal e(t) by taking the difference between the SP and the PV. Op amp "B" is used to invert the signal to the correct polarity before sending it to the Gp(s) simulator. Op amp "B" also will allow RC networks to be inserted to implement lead, lag, and lead/lag compensators or it can be used to implement any other transfer function by selecting the appropriate Zi and Zf. Op amps "C", "D", "E", and "F" simulate Gp(s).

A toggle switch selects the desired system type and places the correct PV on the Output jack.

The simulation parameters $\mathrm{k}, \mathrm{a}$, and $\mathrm{b}$ are set by adjusting the appropriate 10k pots. The theoretical range of parameter variation with $10 \mathrm{k}$ pots is from 1.0 to 20.0 ; however, because of pot sensitivity, the practical range is 1.0 to 10.0. The parameter value depends on the value of the feedback capacitor $\mathrm{Cf}$ and the $10 \mathrm{k}$ pot setting. Since high tolerance capacitors are fairly expensive, a capacitor of $100 \mathrm{uF}$ or slightly above can be used, and the pot adjusted to achieve the desired parameter value using the expression.

$$
\mathrm{R}=1 / \mathrm{Cf} * \text { parameter value }
$$

The simulator is also equipped with a second a second set of 100k pots (labeled .a and .b on the circuit board) so parameters $\mathrm{a}$ and $\mathrm{b}$ can also be varied over the range of 0.1 to 1.0. These pots are used by moving the appropriate connecting wire from the $10 \mathrm{k}$ pot to the $100 \mathrm{k}$ pot.

Once $\mathrm{Cf}$ is selected and its value measured, a table similar to Table 1 should be constructed to assist in setting the simulation parameters. 
Table 1. Pot Settings to Achieve Parameters When Cf $=108 \mathrm{uF}$

\begin{tabular}{ccccc}
$\begin{array}{c}\text { 10k pot } \\
\text { Parameter }\end{array}$ & \multicolumn{2}{c}{$\begin{array}{c}\text { 100k pot } \\
\text { Parameter }\end{array}$} & $\frac{\mathrm{R}}{92 \mathrm{k}}$ \\
\cline { 1 - 3 } 1 & $9.2 \mathrm{k}$ & 0.1 & \\
2 & $4.6 \mathrm{k}$ & 0.2 & $46 \mathrm{k}$ \\
3 & $3.1 \mathrm{k}$ & 0.3 & $31 \mathrm{k}$ \\
4 & $2.3 \mathrm{k}$ & 0.4 & $23 \mathrm{k}$ \\
5 & $1.8 \mathrm{k}$ & 0.5 & $18 \mathrm{k}$ \\
6 & $1.5 \mathrm{k}$ & 0.6 & $15 \mathrm{k}$ \\
7 & $1.3 \mathrm{k}$ & 0.7 & $13 \mathrm{k}$ \\
8 & $1.1 \mathrm{k}$ & 0.8 & $11 \mathrm{k}$ \\
9 & $1.0 \mathrm{k}$ & 0.9 & $10 \mathrm{k}$ \\
10 & $.92 \mathrm{k}$ & 1.0 & $9.2 \mathrm{k}$ \\
15 & $.62 \mathrm{k}$ & 1.5 & $6.2 \mathrm{k}$ \\
20 & $.46 \mathrm{k}$ & &
\end{tabular}

The overall gain of op amps " $D$ " and " $F$ " must be 1 . Once feedback capacitors have been selected and measured, the input resistors must be set using the expression

$$
\mathrm{R}=1 / \mathrm{C}
$$

Resistor(s) or an appropriately sized pot can be used.

For various control concepts it is desirable to observe the system response to a step input. The circuit shown in Figure 4 was added to provide a step change from 2 volts to 3 volts. The input to the circuit is connected to the $-15 \mathrm{~V}$ supply and the output is connected to the simulator input jack. While the switch across the $1 \mathrm{k}$ resistor is closed, the circuit provides a 2 Volt input to the simulator. When the switch is opened, the output of the circuit changes to 3 Volts, thereby providing a step change to the input of the simulator.

\section{Sample Experiments}

The following experiments illustrate several concepts that can be demonstrated using the simulator. A number of other concepts and plant transfer function demonstration are also possible.

For each of the following experiments, the desired $\mathrm{Gp}(\mathrm{s})$ is established by setting the toggle switch to the appropriate system type and varying the appropriate pots in accordance with Table 1 to set parameters $\mathrm{k}$, a, and $\mathrm{b}$. 


\section{Experiment \#1 - Effects of Damping Ratio}

Damping ratio effects can be illustrated by setting the simulator for system type 0 , parameter $\mathrm{k}$ to 1 and parameter $b$ to 4 (damped natural frequency of 2 radians/second). This corresponds to a plant transfer function of

$$
\mathrm{Gp}(\mathrm{s})=1 /\left(\mathrm{s}^{\wedge} 2+\mathrm{as}+4\right)
$$

Varying parameter a from 0.3 to 6.0 and applying a set change from 2 Volts to 3 Volts at the input shows the response differences between under damped, critically damped, and over damped. If the output is plotted on a stripchart recorder or $x-y$ plotter, percent overshoot, frequency of oscillation, etc. are easily compared to theoretical values.

\section{Experiment \#2 - System Stability}

System stability can be demonstrated by setting the system type to 1 , parameter a to 0.5 and parameter $b$ to 8 . This gives

$$
\mathrm{Gp}(\mathrm{s})=\mathrm{k} / \mathrm{s}\left(\mathrm{s}^{\wedge} 2+0.5 \mathrm{~s}+8\right)
$$

Gradually increasing parameter $\mathrm{k}$ from 1 will cause the system to go unstable. Simulation results are easily compared with those obtained using Routh-Hurwitz and/or other methods.

\section{Experiment \#3 - Steady State Error}

Steady state error can be demonstrated by setting the system to type 0 , parameter $\mathrm{k}$ to 1 , parameter a to 4 and parameter $b$ to 5 . This gives

$$
\mathrm{Gp}(\mathrm{s})=1 /\left(\mathrm{s}^{\wedge} 2+4 \mathrm{~s}+5\right)
$$

Variations in steady state error can be monitored as the SP is varied from 2 Volts and 3 Volts. The system type can then be changed to 1 and the steady state error is zero regardless of the value of the SP.

\section{Experiment \#4 - Compensators}

A variety of compensator experiments can be run. The compensators can be designed using the root locus method or the frequency domain method and then implemented as a RC network between op amps A and B. alternatively, Zi and Zf can be selected to achieve the desired compensator transfer function.

The following example illustrates a lag compensator designed using root locus methods. Before the lab, the students are given the following instructions:

Given the plant transfer function

$$
\mathrm{Gp}(\mathrm{s})=\mathrm{Ku} / \mathrm{s}\left(\mathrm{s}^{\wedge} 2+4 \mathrm{~s}+5\right)
$$

Calculate the uncompensated gain Ku to give a damping ratio of 0.7. (The students should calculate a value near 3). Round the calculated value of $\mathrm{Ku}$ to the nearest whole integer and then 
design a lag compensator to improve the error coefficient by a factor of 10. (The students should find a compensator with a transfer function of

$$
\operatorname{Gc}(s)=(3 s+6) /(s+0.2)
$$

In the lab, the students set the simulator for the uncompensated system and plot the output for a setpoint change from 2 Volts to 3 Volts. The lag compensator is implemented by making $\mathrm{Zi}$ a 5 $\mathrm{k}$ resistor in parallel with a $100 \mathrm{uF}$ capacitor and by making $\mathrm{Zf}$ a $150 \mathrm{k}$ resistor in parallel with a $33 \mathrm{uF}$ capacitor. The output of the compensated system is then plotted for a setpoint change from 2 Volts to 3 Volts. This plot of the compensated system is compared to the uncompensated system to show that the steady state error is improved while relative stability is decreased.

\section{Experiment \#5 - Root Locus}

This experiment shows how the gain $\mathrm{k}$ affects the roots of the characteristic equation and how the roots correspond to system response. The simulator is set for

$$
\mathrm{Gp}(\mathrm{s})=\mathrm{k} / \mathrm{s}\left(\mathrm{s}^{\wedge} 2+4 \mathrm{~s}+5\right)
$$

Matlab (or other simulator) is used to plot the root locus of $\mathrm{Gp}(\mathrm{s})$ and to find the roots at various values of $\mathrm{k}$. The simulator is then run for each $\mathrm{k}$ and the damped frequency is measured. The simulator results are compared with the roots from Matlab. Observations regarding the damping ratio and relative stability can also be made.

Experiment \#6 - PID Controllers

Characteristics of PID control can be demonstrated by setting the simulator for a plant transfer function of

$$
\mathrm{Gp}(\mathrm{s})=1 / \mathrm{s}\left(\mathrm{s}^{\wedge} 2+2 \mathrm{~s}+4\right)
$$

and obtaining a response to a step input.

$\mathrm{Gp}(\mathrm{s})$ is then isolated by disconnecting the output of op amp B from the pot corresponding the parameter $\mathrm{k}$. If this is done by lifting the wire from the breadboard at op amp B, the "liftedwire" becomes the input to $\mathrm{Gp}(\mathrm{s})$. The open loop system response for a step change from 2 Volts to 3 Volts is then plotted. The dead time, reaction time, and system gain are obtained from this plot, and the parameters for a P controller, PI controller, and PID controller are calculated.

Either a commercially available PID controller or a PLC can be used to implement each of the controllers. The results from a step input for each of the different types of controllers are then compared to the response without the controller. The simulator parameters can also be changed to demonstrate situations that are best suited for each type of controller.

\section{Experiment \#7 - Gain Margin}

Gain margin effects can be demonstrated by setting the simulator for a plant transfer function of 


$$
\mathrm{Gp}(\mathrm{s})=1 / \mathrm{s}\left(\mathrm{s}^{\wedge} 2+.5 \mathrm{~s}+4\right)
$$

This system has a gain margin of approximated $6 \mathrm{~dB}$ and this corresponds to a gain of 2 . The value of parameter $\mathrm{k}$ is gradually increased until the response to a step input causes instability to occur.

The simulator's operation was compared to Matlab simulations for these experiments. Final voltage values compared exactly. Voltage extremes for under damped responses were within 5\% as were frequencies of oscillation.

It is possible that certain parameter settings can cause one or more of the op amps to saturate. Therefore, before an experiment is run, it should be simulated on Electronic Workbench (or other simulator) to ensure that none of the op amps go into saturation.

\section{Cost}

If all the components are purchased new, the simulator can be built for around $\$ 15$. However, most of the components are usually already on the shelves of most electrical engineering labs and so the cash outlay could be very minimal.

\section{Conclusion}

This paper has described an inexpensive simulator that can be easily used to demonstrate various concepts in feedback control. The basis for the circuit operation was developed and some implementation notes were given. Example lab experiments were described to show the flexibility of the simulator and to provide a basis for developing further experiments. 


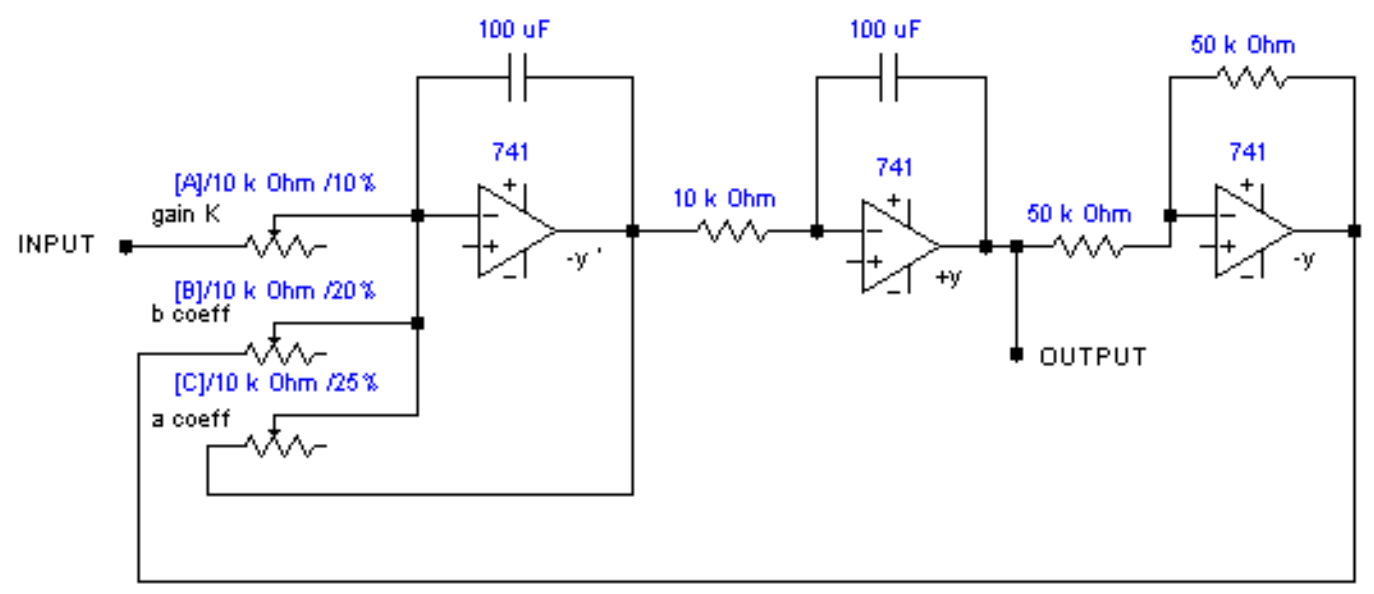

Figure 1. Type 0 System Simulator

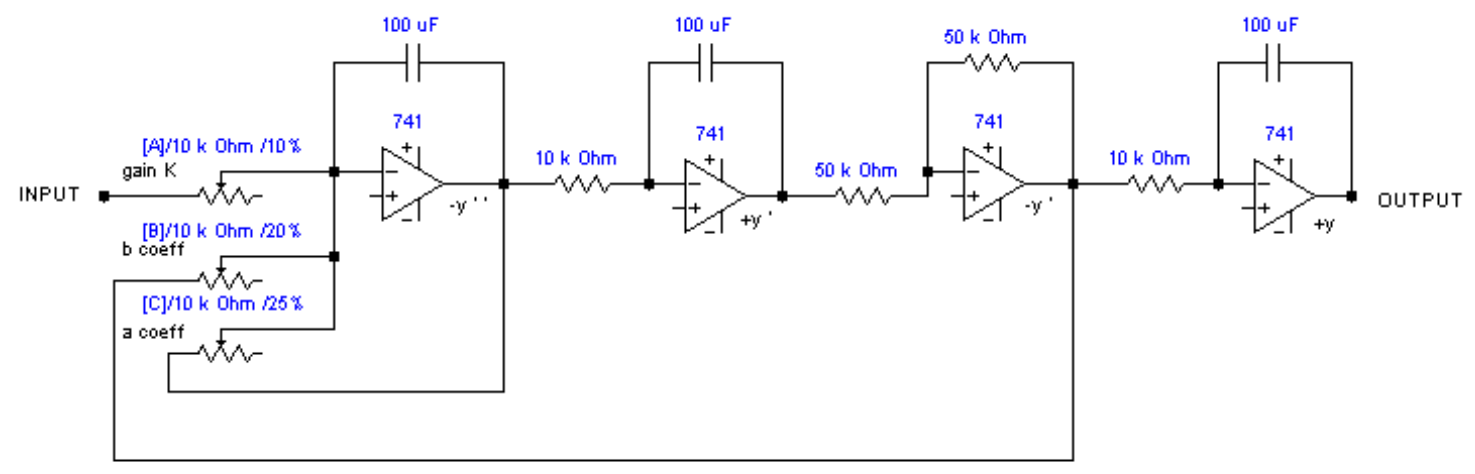

Figure 2. Type 1 System Simulator

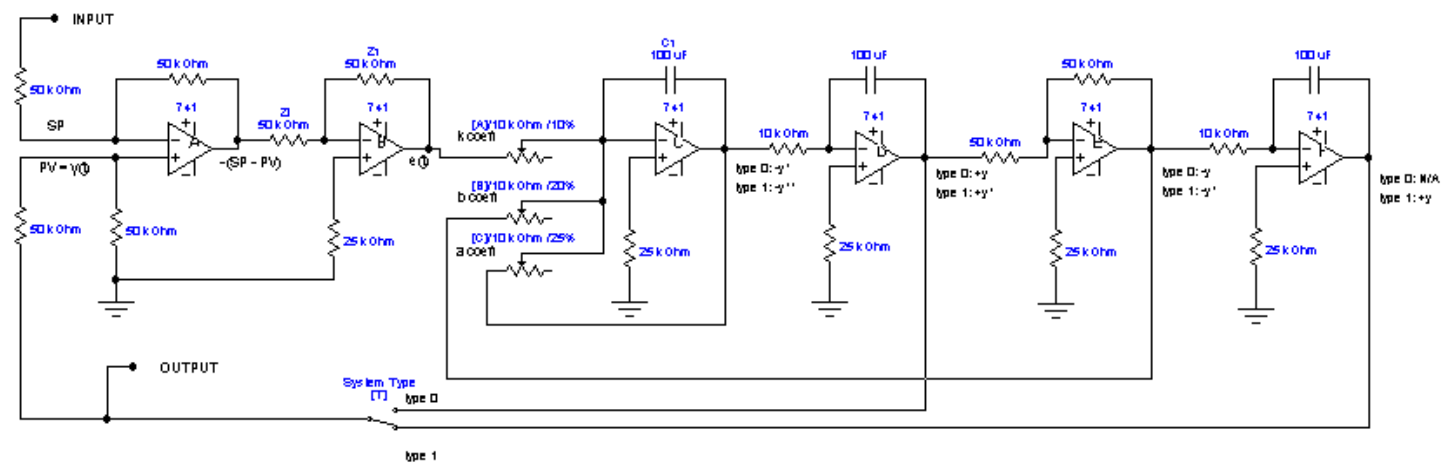

Figure 3. Control System Simulator 


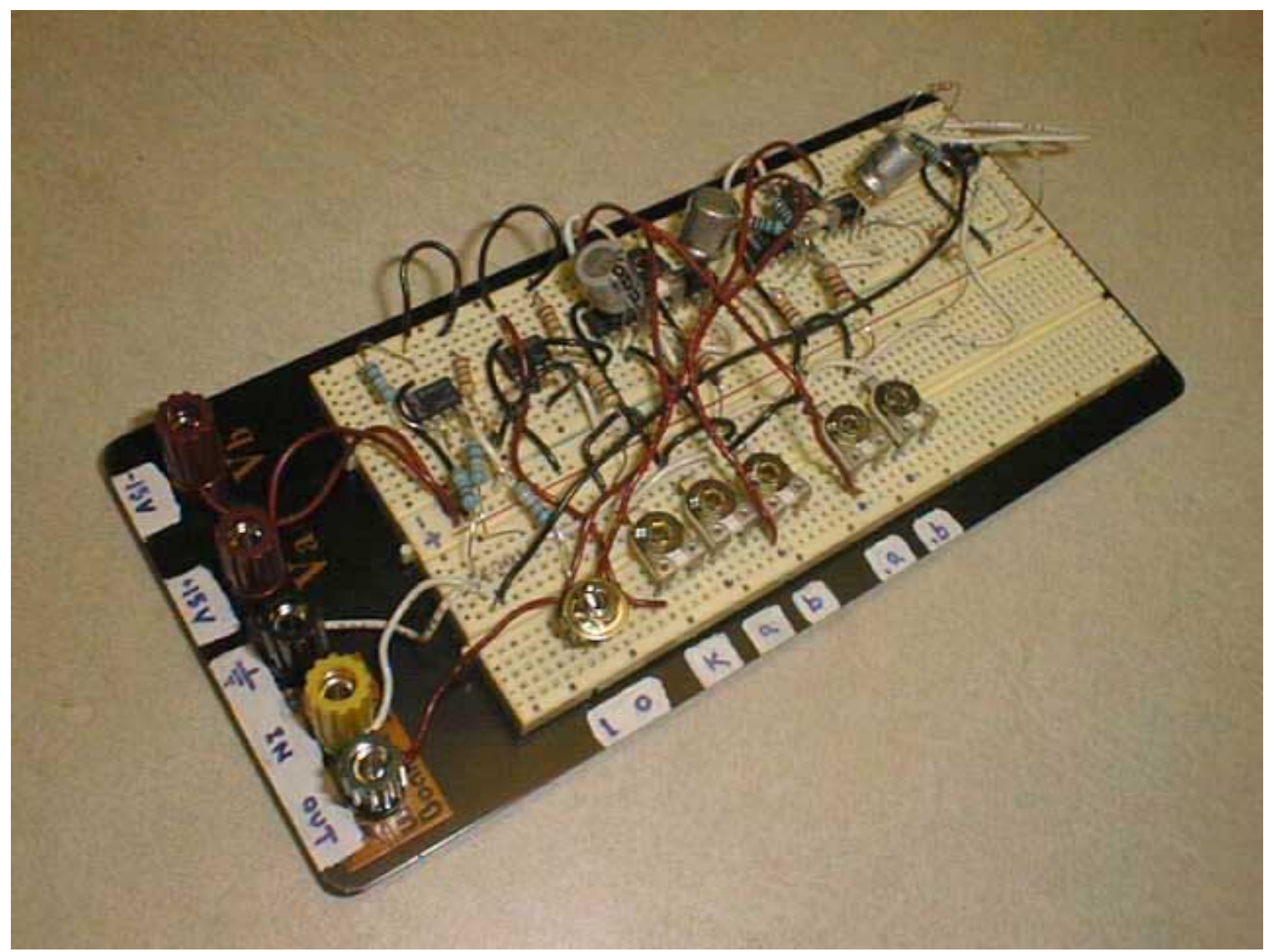

Figure 4. System Simulator

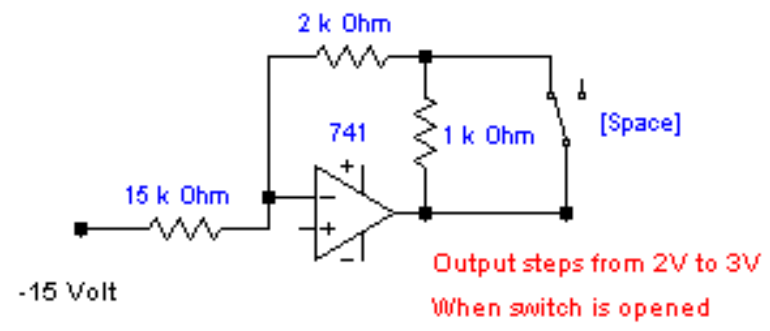

Figure 5. Step Function Generator 
ROBERT LYNN MUELLER

Robert Lynn Mueller is an assistant professor of Electro-Mechanical Engineering at the New Kensington Campus of the Pennsylvania State University. He received his B.S. in Electrical Engineering from Wichita State University and his Ph.D. in Electrical Engineering from the University of Pittsburgh. Dr. Mueller spent 30 years working with industrial control systems before joining Penn State and still continues to work as a consultant providing advice regarding industrial automation. 